Calcaneal Tendon Collagen Fiber Morphometry and Aging

Peer-reviewed author version

HADRABA, Daniel; Janacek, Jiri; Filova, Eva; Lopot, Frantisek; PAESEN, Rik; Fanta, Ondrej; Jarman, Anneliese; Necas, Alois; AMELOOT, Marcel \& Jelen, Karel (2017)

Calcaneal Tendon Collagen Fiber Morphometry and Aging. In: MICROSCOPY AND MICROANALYSIS, 23(5), p. 1040-1047.

DOI: $10.1017 / S 1431927617012569$

Handle: http://hdl.handle.net/1942/25491 


\title{
Calcaneal tendon collagen fibre morphometry and aging
}

\author{
Daniel Hadraba, ${ }^{1,2,3 *}$ Jiri Janacek, ${ }^{1}$ Eva Filova, ${ }^{4}$ Frantisek Lopot, ${ }^{2}$ Rik Paesen, ${ }^{3}$ Ondrej \\ Fanta, ${ }^{2}$ Anneliese Jarman, ${ }^{5}$ Alois Necas, ${ }^{6}$ Marcel Ameloot, ${ }^{3}$ Karel Jelen ${ }^{2}$
}

1 - Department of Biomathematics, Institute of Physiology, The Czech Academy of Sciences, Videnska 1083, Prague 4,

Czech Republic, 14220

2 - Department of Anatomy and Biomechanics, Faculty of Physical Education and Sport, Charles University, Jose Martiho

31, Prague 6, Czech Republic, 16200

3 - Department of Biophysics, Biomedical Research Institute, Hasselt University, Agoralaan building C, Diepenbeek,

Belgium, B-3590

4 - Department of Tissue Engineering, Institute of Experimental Medicine, The Czech Academy of Sciences, Videnska 1083, Prague 4, Czech Republic, 14220

5 - Department of Tissue Engineering \& Biophotonics, King's College London, Guy's Campus, Great Maze Pond, London, United Kingdom, SE1 9RT

6 - Faculty of Veterinary Medicine, University of Veterinary and Pharmaceutical Sciences Brno, Palackeho tr. 1/3, Brno,

Czech Republic, 61242

*Correspondence to: $\underline{\text { daniel.hadraba@fgu.cas.cz }}$

\begin{abstract}
Fibrillar collagen in tendons and its natural development in rabbits are discussed in this paper. Achilles tendons from newborn ( 7 days) to elderly ( $\sim 38$ months) rabbits were monitored in intact $\left(\mathrm{n}_{\text {tendons }}=24\right)$ and microtome sectioned $\left(\mathrm{n}_{\text {tendons }}=11\right)$ states with label-free SHG microscopy. After sectioning, the collagen fibre pattern was irregular for the younger animals and remained oriented in parallel to the load axis of the tendon for the older animals. In contrast, the collagen fibre pattern in the intact samples followed the load axis for all the age groups. However, there was significant difference in the tendon crimp pattern appearance between the age groups. The crimp amplitude $(A)$ and wavelength $(\Lambda)$ started at very low values $(A=2.0 \pm 0.6 \mu \mathrm{m}, \Lambda=19 \pm 4 \mu \mathrm{m})$ for the newborn animals. Both parameters increased for the sexually mature animals $(>5$ months old). When the animals were fully mature the amplitude decreased but the wavelength kept increasing. The results revealed that the microtome sectioning artefacts depend on the age of animals and that the collagen crimp pattern reflects the physical growth and development.
\end{abstract}

Keywords collagen, aging, crimp, fibre orientation, and tendon. 


\section{Introduction}

The hierarchical architecture of tendon consists of collagen molecules which form fibrils, fibre bundles and fascicles (Wang, 2006). The structural arrangement remodels with disease, diet, physical activity, or age at each hierarchical level (Clemmer, et al., 2010; Holzapfel, 2008; Svensson, et al., 2013). At the fibre level the major arrangement, which zig-zags, following the longitudinal load axis (Maeda, et al., 2007), is termed the crimp pattern. It has been proposed that the crimp pattern is linked to the contraction of tendon fibroblasts (Herchenhan, et al., 2012), or to a sudden twist in the direction of fibrils that is marked by nanoscopic knots/hinges (Franchi, et al., 2010), but no dedicated structure was found for the knot/hinge region (Raspanti, et al., 2005). In general, the crimp pattern is represented by two parameters; length and angle/height (Legerlotz, et al., 2014; Patterson-Kane, et al., 1997). These parameters often vary among different studies depending on species, anatomical location, strain state, loading history or tendon region (Hansen, et al., 2002; Legerlotz, et al., 2014; Wang, et al., 2012; Zhao, et al., 2015). Commonly a crimp pattern oscillates with a periodicity between $10 \mu \mathrm{m}$ and $100 \mu \mathrm{m}$ whilst the variability in the crimp height is much lower $(\sim 5 \mu \mathrm{m})$ (Freed \& Doehring, 2005). Certain disagreement also appears among studies which compare the crimp length and height/angle with respect to aging. For instance, Patterson-Kane et al. published that both the crimp length and angle had a decreasing tendency with age in the central region of superficial digital flexor tendon (Patterson-Kane, et al., 1997). On the other hand Legerlotz et al. stated that the crimp height and length development with age was parabolic (Legerlotz, et al., 2014). These results confirm some heterogeneity among the studies and consequent discussion about the factors which influence the crimp pattern parameters and development, for instance cell-matrix interactions, exercise or increased number of cross-links. Not only is development of the crimp pattern still a subject of discussion but its function also is (Raspanti, et al., 2015). The most frequent suggestion for the function of the crimp pattern is that it acts as a mechanical buffer (Franchi, et al., 2007).

To image the crimp pattern, polarization light microscopy is generally favoured as collagen fibres are birefringent (Vidal, 2003). The orientation of collagen fibres is calculated from images acquired under different polarization angles (Dickey, et al., 1998; Thomopoulos, et al., 2006). Other conventional imaging methods frequently utilized for imaging collagen fibres include widefield microscopy with histochemical or immunochemical staining. However, these methods are often restricted to superficial imaging because of light scattering 
(Ntziachristos, 2010) or limited penetration depth of labels (Melvin \& Sutherland, 2010). To overcome these limitations, the sample undergoes multi-step preparation including sectioning, staining, rinsing, (de)hydrating, or fixation with chemicals before data acquisition. Then the orientation of collagen fibres is retrieved from the images using techniques such as the gradients of image intensity (Courtney, et al., 2006; Karlon, et al., 1998), fractals (Thomason, et al., 1996), Hough transform (Bayan, et al., 2009), Fourier transform (Sivaguru, et al., 2010), Gabor transform (Osman, et al., 2013) or Wavelet transform (Castellano, et al., 2004), or their combination (Frisch, et al., 2012). Despite the quality of these algorithms, the multi-step preparation can lead to biased results (Carlo \& Stevens, 2011; Dorph-Petersen, et al., 2001; Janacek, et al., 2012; Park, et al., 2015).

Unlike these conventional imaging methods, second harmonic generation (SHG) microscopy does not require destructive multi-step sample preparation (Stoller, et al., 2002). This non-linear label-free qualitative method benefits from the presence of fibril-forming type I collagen and its unique non-centrosymmetric molecular composition (non-zero secondorder susceptibility tensor $\chi^{(2)}$ (Pavone \& Campagnola, 2013; Rocha-Mendoza, et al., 2007; $\mathrm{Su}$, et al., 2011)) (Myllyharju \& Kivirikko, 2004). This composition generates the frequency-doubled SHG signal in the sample when interacting with the electric field. The intensity of the signal does not depend only on the strength of the electric driving field, but also on the density of the molecule and on the orientation of the molecule axis relative to the polarization state of the incident laser beam (Cicchi, et al., 2013). As a short-pulse near infrared laser beam is used, the imaging depth is significantly extended (Deniset-Besseau, et al., 2009; Zhuo, et al., 2007) and consequently, the observed intact volume becomes larger, and the photodamage to the sample becomes smaller (Chen, et al., 2012a).

To enrich the existing knowledge in sample processing and connective tissue aging, this study presents two elementary challenges. (1) First, it investigates the impact of sample microtome sectioning on the interpretation of the fibre morphology with respect to aging and it also searches for an efficient, quantitative technique to determine the orientation regularity (changes in orientation of fibres with distance in longitudinal plane) from a single image. (2) Second, it examines the trends in the development of the collagen crimp pattern in samples of the Achilles tendon of rabbits from birth to elderly and compares these results with the images acquired from microtome sections. 


\section{Methods}

\subsection{Tendon subjects: sections and intact samples}

The rabbit Achilles tendon (tendo calcaneus communis) served as model connective tissue because of its high content of parallel type I collagen fibres and explicit in vivo load directionality. The group of animals consisted of New Zealand white, Czech Spot and Belgian Hare crossbred rabbits. The animals were bred in hutches and originated from different litters. The legs were received as animal waste. The animals were selected to represent the main developmental stages, newborn (up to 6 weeks), sexually mature (5 to 7 months) and elderly (up to 5 - 7 years) (Houdebine \& Fan, 2009; Varga, 2013). The tendons were dissected from both hind legs. The tendon samples were left intact (zero-load state, 24 tendons from 13 animals between 7 days and 38 months) or mounted with Cryomount (Histolab Products AB, Sweden), cryocut into $20 \mu \mathrm{m}$ sections (zero-stress state, 11 tendons from 6 animals between 4 weeks and 37 months) with the Leica CM1850 cryostat (Leica, Germany) and covered with phosphate-buffered saline. The microtome sections were performed in steps of $100 \mu \mathrm{m}$ along the approximately $1 \mathrm{~mm}$ depth from the tendon's surface.

\subsection{Tendon imaging using SHG microscopy}

The microscope image acquisition was performed on two independent systems in order to increase the robustness of the investigation. The first system (System I) consisted of a confocal scanning head (Leica TCS SP2 AOBS, Leica, Germany) mounted to an inverted microscope (DMIRE2, Leica, Germany) and coupled with a femtosecond tunable pulse laser (Ti:Sapphire Chameleon Ultra, Coherent Inc., USA). The laser was set to $860 \mathrm{~nm}$ and the horizontally polarized incident beam reached the sample through the objective (HC PL APO 20x/0.75 IMM, image resolution 1024x1024, pixel size $732 \mathrm{~nm}$ or HCX PL APO 63x/1.2 W, image resolution 2048x2048, pixel size $51 \mathrm{~nm}$, Leica, Germany). The detection of the SHG signal was in backwards, non-descanned mode. To guarantee the SHG selectivity, an IR blocking filter (703HSP, Laser Components GmbH, Germany) and a single band-pass filter (HQ430/20 M-2P, Chroma, USA) were placed before a photomultiplier tube (PMT) detector.

The second system (System II) consisted of a confocal scanning microscope (Zeiss LSM 510 META, Zeiss, Germany) linked to a motorized inverted Axiovert 200M (Zeiss, Germany) and coupled with a femtosecond tunable pulse laser (Mai Tai DeepSee, Spectra- 
Physics, USA). The $810 \mathrm{~nm}$ horizontally-polarized incident beam entered the samples through an objective (Plan-Apo 20x/0.75 dry, image resolution 2048x2048, pixel size $220 \mathrm{~nm}$, Zeiss, Germany). In the emission pathway, the light was collected in the forward direction passing through an in-house built condenser (adjustable numerical aperture 0 to 0.8), the appropriate set of filters (FT 442, Zeiss, Germany and BP 405/10, Semrock, USA) and entering a multi-alkali PMT detector.

The samples were placed on microscope cover slips, moistened with phosphate-buffered saline and left uncovered to prevent deformation. The microtome sections from each tendon were optically scanned by System I in the central $\mathrm{Z}$ depth at up to three randomly selected positions. The intact samples were scanned by System I or System II in the central XY position of the tendon from the top to an average depth of $100 \mu \mathrm{m}$.

\subsection{Fibre orientation analysis in microtome sections}

The structural organization of collagen in the unstained microtome sections was quantified through the fibre orientation regularity. In this approach, the fibre orientation was given by the local tensor representation (Jähne, 2005). First, the image was scanned with a $16 \times 16$ pixel sliding window that moved over the image coordinates $x, y$ with an 8-pixel step. Each $16 \times 16$ pixel window-cropped image was transformed into the frequency domain by Fast Fourier Transform (FFT). The major peak $G_{i, j}$ of the centred FFT modulus $\left|F_{i, j}\right|$ was established using reconstruction by dilation of the modulus $\left|F_{i, j}\right|$ around the central pixel (Soille, 2013). The structure tensor $J_{\xi, \eta}$ was calculated from the $2^{\text {nd }}$ order moments

$$
J_{\xi, \eta}=\left[\begin{array}{cc}
\sum_{i, j} G_{i, j}(i-\bar{\imath})^{2} & \sum_{i, j} G_{i, j}(i-\bar{\imath})(j-\bar{\jmath}) \\
\sum_{i, j} G_{i, j}(j-\bar{\jmath})(i-\bar{\imath}) & \sum_{i, j} G_{i, j}(j-\bar{\jmath})^{2}
\end{array}\right]=\left[\begin{array}{cc}
J_{11} & J_{12} \\
J_{21} & J_{22}
\end{array}\right],
$$

where $1 \leq i \leq 16,1 \leq j \leq 16, \bar{\imath}=\bar{J}=8.5, J_{12}=J_{21}$.

This gives a $2 \times 2$ symmetric tensor $J_{\xi, \eta}$ for each rescaled pixel $\xi, \eta$ where $\xi=\frac{x}{8}, \eta=\frac{y}{8}$. Indirectly, the matrix defines the values for hue, saturation and value (HSV) colour format. Inspired by Jähne (Jähne, 2005), the hue $2 \varphi$ was calculated by rotating the structure tensor into the canonical coordinate system by angle $-\varphi$. The matrix multiplication yields the pixel orientation angle $\varphi$ as

$$
\tan 2 \varphi_{\xi, \eta}=\frac{2 J_{12}}{J_{22}-J_{11}}, \text { where } \varphi \in<0, \pi>
$$


the value $v$ (pixel brightness) as the trace of the tensor,

$$
v_{\xi, \eta}=J_{11}+J_{22},
$$

and the saturation $s$ as coherence calculated from $J_{11}, J_{12}, J_{22}$ as

$$
S_{\xi, \eta}=\frac{\sqrt{\left(J_{11}-J_{22}\right)^{2}+4 J_{12}^{2}}}{J_{11}+J_{22}}
$$

Finally, the HSV image is converted to red-green-blue (RGB) format, the changes in colour and intensity represent the orientation irregularity.

To interpret the data objectively, the fibre organization in the image was computed through an autocorrelation approach using the aforementioned spatial variables: orientation angle $\varphi_{\xi, \eta}$, value $v_{\xi, \eta}$, and saturation $s_{\xi, \eta}$. First, the complex value $p_{\xi, \eta}$ for each pixel is calculated as a combination of trigonometric functions

$$
p_{\xi, \eta}=v_{\xi, \eta} s_{\xi, \eta}\left(\cos 2 \varphi_{\xi, \eta}+\boldsymbol{i} \sin 2 \varphi_{\xi, \eta}\right)
$$

where $\boldsymbol{i}$ is the imaginary unit. Then the complex $c_{k, l}$ and modulus $r_{k, l}$ autocorrelation functions for $p_{\xi, \eta}$ were calculated as

$$
c_{k, l}=\sum_{r=0}^{R-1} \sum_{s=0}^{S-1} p_{r, s} \bar{p}_{r+k, s+l}
$$

and

$$
r_{k, l}=\sum_{r=0}^{R-1} \sum_{S=0}^{S-1}\left|p_{r, s}\right|\left|p_{r+k, s+l}\right|
$$

where $0<k \leq R-1,0<l \leq S-1, \quad \bar{p}$ denotes the complex conjugate of $p,\left|p_{r, s}\right|$ and $\left|p_{r+k, s+l}\right|$ the moduli, and $p_{r, s}=0$ if $\xi>R-1$ or $\eta>S-1$ for the zero padded $p_{r, s}$.

In the next step, the distances in the image were sectioned into 32 equal annular intervals by $\Delta d=\frac{\text { xsize of the image }}{32}$. The returned autocorrelation ratios $\frac{\left|c_{k, l}\right|}{r_{k, l}}$ were subscribed to these relevant intervals according to distance $d=\sqrt{i^{2}+j^{2}}$ where $d_{m} \leq d<d_{m+1}$. The resulting values for the correlogram $C\left(d_{m}\right)$, where $m$ is the interval/bin number, were calculated as the average values of the $n_{m}$ ratios $\frac{\left|c_{k, l}\right|}{r_{k, l}}$ in each $d_{m}$, i.e.

$$
C\left(d_{m}\right)=\frac{1}{n_{m}} \sum_{q=1}^{n_{m}}\left(\frac{\left|c_{k, l}\right|}{r_{k, l}}\right)_{q}
$$


The $\frac{\left|c_{k, l}\right|}{r_{k, l}}$ ratio was used to emphasize the role of orientations in autocorrelations. For a perfectly oriented isotropic pattern, $C\left(d_{m}\right)$ equals 1 . The resulting graphs were fitted with an exponential model $y=B e^{-C x}+D$ where $B, C$, and $D$ are freely adjustable parameters. The coefficient of determination was calculated, and the residual plot was performed. Models that explained more than $90 \%$ of the variability of the data were subjected to further analysis.

\subsection{Crimp analysis and comparison with microtome sections}

In the intact samples, the crimp amplitudes and wavelengths were extracted manually (Fig. 1).

Based on this manual measurement, the mean crimp amplitude and wavelength were stated for each age group, and the results were simulated in a grayscale value image $I_{x, y}$ using

$$
I_{x, y}=\sin \left(2 \pi F\left(x+A \sin \left(\frac{2 \pi y}{\Lambda}\right)\right)\right)
$$

where $x, y$ are the coordinates of the image, $F$ defines the thickness of the fibres, $\Lambda$ is the measured wavelength of the crimp pattern, and $A$ stands for the measured amplitude of the crimp pattern. After that, these simulated grayscale value images were analysed by the same algorithm as the images of the microtome sections. The results were again expressed in correlograms. Finally, the correlograms for the simulated images of the intact samples $C_{\text {sim }}$ and microtome sections $C_{\text {sec }}$ were compared by mean percentage difference (MPD)

$$
M P D=\frac{1}{n} \sum_{d=1}^{n} \frac{\left|C_{s e c}(d)-C_{s i m}(d)\right|}{\left(C_{s e c}(d)+C_{s i m}(d)\right) / 2} \times 100,
$$

where $n=32$ bins.

\subsection{Statistical analysis}

The exponential models of microtome sections, which quantify the fibre orientation regularity, were grouped by age and displayed in a box-and-whisker plot (Fig. 3(b)). The plot represents the relationship between age and the orientation regularity after microtome sectioning. The minimum number of sections per tendon was three, the maximum was nine.

The crimp patterns were analysed through the Z-stacks. The measured parameters (amplitudes and wavelengths) were assigned to the three main developmental stages, checked for normality (S-W test, Q-Q plot) and then compared using a One-way ANOVA followed by 
a post-hoc Tukey's HSD test (Little, 2013). The number of tendons in each developmental stage was; 9 for age $<1$ month, 9 for age 1 month -12 months, and 6 for age $>12$ months (Table 1).

If not stated otherwise, the results are denoted as means with sample standard deviations. The significant value for rejecting the null hypothesis was set to $p$-value $<0.05$. The data analysis was performed in Microsoft Visual C++ 2013 (Microsoft, USA) and Matlab 2015a (Mathworks, USA). The statistical evaluations were performed by using Matlab 2015a.

\section{Results}

\subsection{Unstained microtome sections and orientation regularity analysis}

The images of the unstained microtome sections indicated that the collagen structural organization is age dependent (Fig. 2 - top row). This assumption was confirmed by the analysis of the images and displayed by means of their consequent pseudo-colour representation. The analysed images from young animals possessed rapid colour changes. In contrast, almost single-colour images were returned for the oldest animals (Fig. 2 - bottom row). The changes in the orientation regularity were also expressed numerically by a converging correlogram graph. The correlograms indicated that the fibres in the microtome sections from the very young animals change direction rapidly with distance and these changes do not follow a periodic, regular crimp pattern. On the other hand, the fibres in microtome sections from the older animals are perfectly aligned with the longitudinal axis of the tendon, i.e. the correlogram value is close to one and does not change with distance (Fig. 3). It is clear that with increasing age the orientation regularity tends towards perfect fibrillar orientation anisotropy (Fig. 3(b)).

\subsection{Crimp pattern development}

The organization of the collagen fibres in the load-free, intact samples showed a 3-D frequency-modulated pattern with clear crystallinity. In contrast to the microtome sections, a highly regular crimp pattern was identified for all the age groups including the youngest (Fig. 4). The crimp pattern development with age is quantified in Table 1. Generally, the crimp pattern amplitude was small in the samples from the 7-day-old animal, $A=2.0 \pm 0.6$ $\mu \mathrm{m}$. The amplitude then increased to a maximum of $A=9.6 \pm 3.3 \mu \mathrm{m}$ when the animal was 4 months old. After that, the crimp amplitude decreased to $A=3.0 \pm 1.1 \mu \mathrm{m}$ which is very small compared to the wavelength for the 38 -month-old animal. The wavelength started at its 
minimum for the young animals. It reached the size of $\Lambda=19 \pm 4 \mu \mathrm{m}$ for the 7-day-old animal and kept increasing steadily to $\Lambda=122 \pm 32 \mu \mathrm{m}$ for the 38 -month-old animal. Due to the low amplitude it was sometimes very difficult, or even impossible, to detect the unbiased crimp pattern for the elderly animals. When the amplitude and wavelength data were assigned to each developmental group ( $<1$ month, 1 month -12 months, $>12$ months) and these groups were statistically compared, significant differences were found between all three groups.

\subsection{Crimp pattern simulation and comparison with microtome sections}

The correlogram for the simulated images decreased gradually with distance and oscillated for the young animals (Fig. 5). When comparing the correlograms based on the crimp pattern simulation and microtome sections by calculating MPD (Section 2.3), the MPD differed by $46.0 \%$ for the 4 -week-old group but only by $4.3 \%$ for 36 -week-old group.

\section{Discussions}

The orientation regularity analysis approach revealed a significant difference between the orientation regularity of collagen fibres in the microtome sections from the young and old animals. Representation with a traditional colour-coded scheme (Gillard, et al., 2009) often reduces the sensitivity for orientation detection (Peeters, et al., 2006) and neglects the dependency of neighbouring pixels. The algorithm presented here respects the coherency of the fibres by adjusting the pixel saturation and brightness accordingly. In addition, the spatial inhomogeneity in the scalar fields was described through autocorrelation functions. To prioritize the orientation over magnitude, the complex autocorrelation function was divided by the modulus (saliency) autocorrelation, and the returned correlogram quantified the anisotropy of the fibres within the images. This approach is fully automated and transferable for detection of the orientation regularity of any fibres imaged at sufficient resolution. Finally, this analysis approach is not limited only to superficial regions because it does not rely on the preservation of polarization state such as the SHG polarization analysis, and therefore does not suffer from the randomization of polarization state caused by scattering in tissue (Chen, et al., 2012b). This is important for analysing in-vivo experiments in which most of the light detected is backscattered.

Unlike the microtome sections, all the intact samples in the load-free state possessed strong orientation regularity of the fibres in the direction of the main load-bearing axis. This shows that microtome sectioning can cause morphological changes in connective tissue. 
Surprisingly, these alterations are highly dependent on the age of the subject, i.e. the younger the animal, the more pronounced the changes, and the higher value MPD reached. The cause of alterations to morphology can be explained through several underlying mechanisms. For example, a different level of residual stresses in the tissue due to the presence of fibril ends which disappear in mature tissue (Provenzano \& Vanderby Jr, 2006) or a lower number of enzymatic and non-enzymatic cross-links (Avery \& Bailey, 2005) at early developmental stages.

All the intact tendons, including those from very young animals, displayed the crimp pattern. However, the parameters of the crimp pattern vary significantly among the intact samples. The amplitude of the crimp pattern exhibited a highly nonlinear dependency on age and reached its maximum at sexual maturity, but the wavelength of the crimp pattern seemed to grow steadily with age. This finding is in contrast to what is reported by Patterson-Kane et al. and Legerlotz et al. (Legerlotz, et al., 2014; Patterson-Kane, et al., 1997). Legerlotz et al. found almost no significant differences in the amplitude of crimps, and a decrease in the wavelength at the end of the observed period (mice from 3 weeks to 6 months). PattersonKane et al. reported an even more dramatic decrease in the wavelength during the observed period (horses from 2 years to 15 years). This contradiction could be caused by several factors, for instance, by the sample source. In the Legerlotz et al. study, the rat tail tendon is not a typical muscle-bone connector in the way a rabbit Achilles tendon is. The Achilles tendon is a functional unit of the musculoskeletal system that must withstand enormous loading. This unit guarantees the most efficient force transfer during ontogeny, and therefore it must react to the muscle volume and power changes (Haus, et al., 2007). Therefore, the suggested role of the crimp pattern as a mechanical buffer (Franchi, et al., 2007) is questionable and, more likely, the crimp pattern has a preload function which decreases the amount of initial loading force from muscles. This ability can be lost as the body movements become limited and/or repetitive with age, and consequently the crimp pattern disappears.

\section{Conclusion}

(1) It has been shown by SHG microscopy that the standard microtome sectioning of tendon causes age-dependent changes to the structure. Therefore, the artefacts caused by sample sectioning or other processing techniques must be considered when interpreting the data, for instance, as a parameter for biomechanical structural models. In addition, a novel approach was successfully adopted to objectively quantify the fibre orientation regularity from a single SHG image. (2) It has been demonstrated that the investigated crimp pattern development is 
not stationary and reflects remodelling and perhaps optimization of the musculoskeletal system over time. Additional insight could be obtained by investigating hierarchical composition of collagen and its crimp structure together with material properties, physical activity regimes and other proteins, for example glycosaminoglycans or cross-linkers.

\section{Acknowledgments}

The project was mainly supported by the Grant Agency of Charles University (grant No. GAUK 956213), Czech Science Foundation No. 16-14758S, by the Ministry of Health of the Czech Republic, grant No. 16-28637A, by the Ministry of Education, Youth and Sports within National Sustainability Programme I, project No. LO1309, by MEYS (LM2015062 Czech-BioImaging), by ERDF OPPK BrainView CZ.2.16/3.1.00/21544, and D.H. acknowledges the support from the Flemish government and Hasselt University for a research stay at Hasselt University.

\section{References}

AVERY, N.C. \& BAILEY, A.J. (2005). Enzymic and non-enzymic cross-linking mechanisms in relation to turnover of collagen: relevance to aging and exercise. Scandinavian Journal of Medicine \& Science in Sports 15(4), 231-240.

Bayan, C., Levitt, J.M., Miller, E., Kaplan, D. \& Georgakoudi, I. (2009). Fully automated, quantitative, noninvasive assessment of collagen fiber content and organization in thick collagen gels. Journal of applied physics 105(10), 102042.

Carlo, C.N. \& Stevens, C.F. (2011). Analysis of differential shrinkage in frozen brain sections and its implications for the use of guard zones in stereology. J Comp Neurol 519(14), 2803-2810.

Castellano, G., Bonilha, L., Li, L.M. \& Cendes, F. (2004). Texture analysis of medical images. Clinical Radiology 59(12), 1061-1069.

Chen, W.L., Hu, P.S., Ghazaryan, A., Chen, S.J., Tsai, T.H. \& Dong, C.Y. (2012a). Quantitative analysis of multiphoton excitation autofluorescence and second harmonic generation imaging for medical diagnosis. Comput Med Imaging Graph 36(7), 519526.

Chen, X., Nadiarynkh, O., Plotnikov, S. \& Campagnola, P.J. (2012b). Second harmonic generation microscopy for quantitative analysis of collagen fibrillar structure. Nat. Protocols 7(4), 654-669. 
Cicchi, R., Vogler, N., Kapsokalyvas, D., Dietzek, B., Popp, J. \& Pavone, F.S. (2013). From molecular structure to tissue architecture: collagen organization probed by SHG microscopy. Journal of Biophotonics 6(2), 129-142.

Clemmer, J., Liao, J., Davis, D., Horstemeyer, M.F. \& Williams, L.N. (2010). A mechanistic study for strain rate sensitivity of rabbit patellar tendon. Journal of Biomechanics 43(14), 2785-2791.

Courtney, T., Sacks, M.S., Stankus, J., Guan, J. \& Wagner, W.R. (2006). Design and analysis of tissue engineering scaffolds that mimic soft tissue mechanical anisotropy. Biomaterials 27(19), 3631-3638.

Deniset-Besseau, A., Duboisset, J., Benichou, E., Hache, F., Brevet, P.-F. \& SchanneKLEIN, M.-C. (2009). Measurement of the Second-Order Hyperpolarizability of the Collagen Triple Helix and Determination of Its Physical Origin. The Journal of Physical Chemistry B 113(40), 13437-13445.

Dickey, J.P., Hewlett, B.R., Dumas, G.A. \& Bednar, D.A. (1998). Measuring Collagen Fiber Orientation: A Two-Dimensional Quantitative Macroscopic Technique. Journal of Biomechanical Engineering 120(4), 537-540.

Dorph-Petersen, K.A., NyengaARD, J.R. \& Gundersen, H.J. (2001). Tissue shrinkage and unbiased stereological estimation of particle number and size. J Microsc 204(Pt 3), 232-246.

Franchi, M., Fini, M., Quaranta, M., De Pasquale, V., Raspanti, M., Giavaresi, G., OtTANi, V. \& RUGGERI, A. (2007). Crimp morphology in relaxed and stretched rat Achilles tendon. Journal of Anatomy 210(1), 1-7.

Franchi, M., Ottani, V., Stagni, R. \& Ruggeri, A. (2010). Tendon and ligament fibrillar crimps give rise to left-handed helices of collagen fibrils in both planar and helical crimps. Journal of Anatomy 216(3), 301-309.

FREED, A.D. \& DoEHRING, T.C. (2005). Elastic model for crimped collagen fibrils. Journal of Biomechanical Engineering 127(4), 587-593.

Frisch, K.E., Duenwald-Kuehl, S.E., LAKES, R.S. \& VANDERBy, R. (2012). Quantification of Collagen Organization Using Fractal Dimensions and Fourier Transforms. Acta Histochemica 114(2), 140-144.

Gillard, J.H., Waldman, A.D. \& BARKeR, P.B. (2009). Clinical MR Neuroimaging: Physiological and Functional Techniques. Cambridge University Press.

Hansen, K.A., Weiss, J.A. \& BARton, J.K. (2002). Recruitment of tendon crimp with applied tensile strain. J Biomech Eng 124(1), 72-77. 
Haus, J.M., Carrithers, J.A., Trappe, S.W. \& TrapPe, T.A. (2007). Collagen, crosslinking, and advanced glycation end products in aging human skeletal muscle. J Appl Physiol 103(6), 2068-2076.

Herchenhan, A., Kalson, N.S., Holmes, D.F., Hill, P., Kadler, K.E. \& Margetts, L. (2012). Tenocyte contraction induces crimp formation in tendon-like tissue. Biomech Model Mechanobiol 11(3-4), 449-459.

HolzAPFEL, G.A. (2008). Collagen in Arterial Walls: Biomechanical Aspects. In Collagen, Fratzl, P. (Ed.), pp. 285-324. Springer US.

Houdebine, L.M. \& FAN, J. (2009). Rabbit Biotechnology: Rabbit genomics, transgenesis, cloning and models. Springer Netherlands.

JÄHNE, B. (2005). Digital Image Processing. Berlin Heidelberg New York: Springer.

JanaceK, J., Kreft, M., Cebasek, V. \& Erzen, I. (2012). Correcting the axial shrinkage of skeletal muscle thick sections visualized by confocal microscopy. J Microsc 246(2), 107-112.

Karlon, W.J., Covell, J.W., McCulloch, A.D., Hunter, J.J. \& Omens, J.H. (1998). Automated measurement of myofiber disarray in transgenic mice with ventricular expression of ras. The Anatomical Record 252(4), 612-625.

Legerlotz, K., Dorn, J., Richter, J., Rausch, M. \& Leupin, O. (2014). Age-dependent regulation of tendon crimp structure, cell length and gap width with strain. Acta Biomater 10(10), 4447-4455.

LitTle, T.D. (2013). The Oxford Handbook of Quantitative Methods, Vol. 2: Statistical Analysis. Oxford, New York: Oxford University Press.

Maeda, E., Shelton, J.C., BAder, D.L. \& Lee, D.A. (2007). Time dependence of cyclic tensile strain on collagen production in tendon fascicles. Biochemical and Biophysical Research Communications 362(2), 399-404.

Melvin, N.R. \& Sutherland, R.J. (2010). Quantitative Caveats of Standard Immunohistochemical Procedures: Implications for Optical Disector-based Designs. Journal of Histochemistry and Cytochemistry 58(7), 577-584.

MyllyharJu, J. \& KIVIRIKKO, K.I. (2004). Collagens, modifying enzymes and their mutations in humans, flies and worms. Trends Genet 20(1), 33-43.

NTZIACHRISTOS, V. (2010). Going deeper than microscopy: the optical imaging frontier in biology. Nat Meth 7(8), 603-614. 
Osman, O.S., Selway, J.L., Harikumar, P.E., Stocker, C.J., Wargent, E.T., CAWThorne, M.A., Jassim, S. \& Langlands, K. (2013). A novel method to assess collagen architecture in skin. BMC Bioinformatics 14(1), 1-10.

PARK, C.Y., LEE, J.K. \& ChUCK, R.S. (2015). Second Harmonic Generation Imaging Analysis of Collagen Arrangement in Human Cornea. Investigative Ophthalmology \& Visual Science 56(9), 5622-5629.

Patterson-Kane, J.C., Firth, E.C., Goodship, A.E. \& Parry, D.A. (1997). Age-related differences in collagen crimp patterns in the superficial digital flexor tendon core region of untrained horses. Aust Vet J 75(1), 39-44.

Pavone, F.S. \& Campagnola, P.J. (2013). Second Harmonic Generation Imaging. Boca Raton, London, NY: CRC Press.

Peeters, T., Vilanova, A., Strijkers, G. \& ter HaAr Romeny, B. Visualization of the fibrous structure of the heart. In Vision, Modeling and Visualization, pp. 309-316.

ProvenZANO, P.P. \& VANDERBy JR, R. (2006). Collagen fibril morphology and organization: Implications for force transmission in ligament and tendon. Matrix Biology 25(2), 7184.

Raspanti, M., Manelli, A., Franchi, M. \& Ruggeri, A. (2005). The 3D structure of crimps in the rat Achilles tendon. Matrix Biology 24(7), 503-507.

Raspanti, M., ReguZzoni, M., Protasoni, M. \& Congiu, T. (2015). Mineralization-related modifications in the calcifying tendons of turkey (Meleagris gallopavo). Micron 71, 46-50.

Rocha-Mendoza, I., Yankelevich, D.R., Wang, M., Reiser, K.M., Frank, C.W. \& Knoesen, A. (2007). Sum Frequency Vibrational Spectroscopy: The Molecular Origins of the Optical Second-Order Nonlinearity of Collagen. Biophysical journal 93(12), 4433-4444.

Sivaguru, M., Durgam, S., Ambekar, R., Luedtke, D., Fried, G., Stewart, A. \& ToussAINT, K.C. (2010). Quantitative analysis of collagen fiber organization in injured tendons using Fourier transform-second harmonic generation imaging. Opt. Express 18(24), 24983-24993.

SoILle, P. (2013). Morphological Image Analysis: Principles and Applications. Berlin Heidelberg: Springer

Stoller, P., Reiser, K.M., Celliers, P.M. \& RubenchiK, A.M. (2002). Polarizationmodulated second harmonic generation in collagen. Biophysical journal 82(6), 33303342. 
Su, P.J., Chen, W.L., Chen, Y.F. \& Dong, C.Y. (2011). Determination of collagen nanostructure from second-order susceptibility tensor analysis. Biophysical journal 100(8), 2053-2062.

Svensson, R.B., Mulder, H., Kovanen, V. \& Magnusson, S.P. (2013). Fracture mechanics of collagen fibrils: influence of natural cross-links. Biophysical journal 104(11), 2476-2484.

Thomason, D.B., Anderson, O. \& Menon, V. (1996). Fractal analysis of cytoskeleton rearrangement in cardiac muscle during head-down tilt. Journal of Applied Physiology 81(4), 1522-1527.

Thomopoulos, S., Marquez, J.P., Weinberger, B., Birman, V. \& Genin, G.M. (2006). Collagen fiber orientation at the tendon to bone insertion and its influence on stress concentrations. Journal of Biomechanics 39(10), 1842-1851.

VARGA, M. (2013). Textbook of Rabbit Medicine. Elsevier Health Sciences UK.

VIDAL, B.D.C. (2003). Image analysis of tendon helical superstructure using interference and polarized light microscopy. Micron 34(8), 423-432.

WANG, J.H.C. (2006). Mechanobiology of tendon. Journal of Biomechanics 39(9), 15631582.

WAnG, J.H.C., GuO, Q. \& LI, B. (2012). Tendon Biomechanics and Mechanobiology-A Minireview of Basic Concepts and Recent Advancements. Journal of Hand Therapy 25(2), 133-141.

Zhao, L., Thambyah, A. \& Broom, N. (2015). Crimp morphology in the ovine anterior cruciate ligament. Journal of Anatomy 226(3), 278-288.

ZHuO, S., CHEN, J., LuO, T., CHEN, H. \& ZHAO, J. High-contrast multimodel nonlinear optical imaging of collagen and elastin. In Journal of Physics: Conference Series, pp. 1476. IOP Publishing. 


\section{Figures}

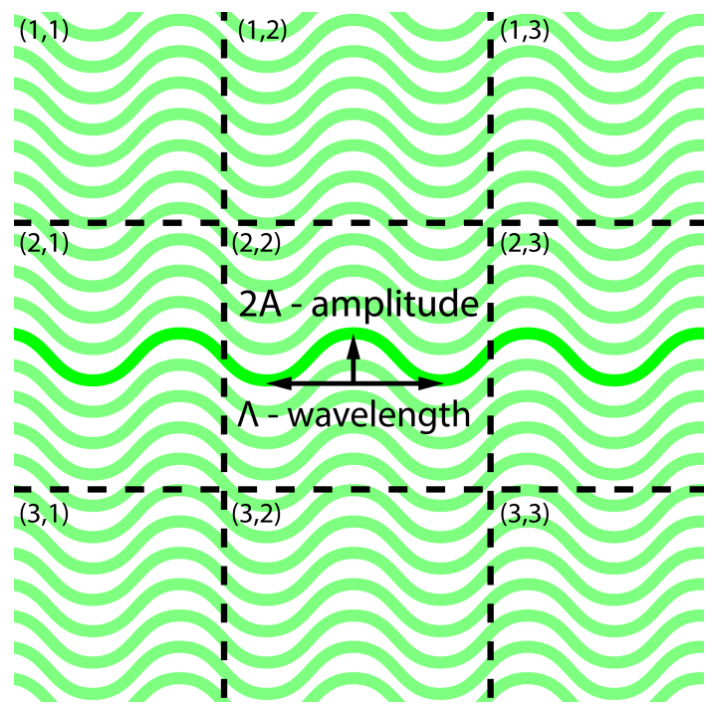

Fig. 1. Manual crimp analysis method. The acquired images were divided into a $3 \times 3$ selection grid. For each field of the grid, crimp amplitudes and wavelengths were calculated from a triangle that connects three adjacent extremes (arrows) of the crimp pattern for a single fibre.

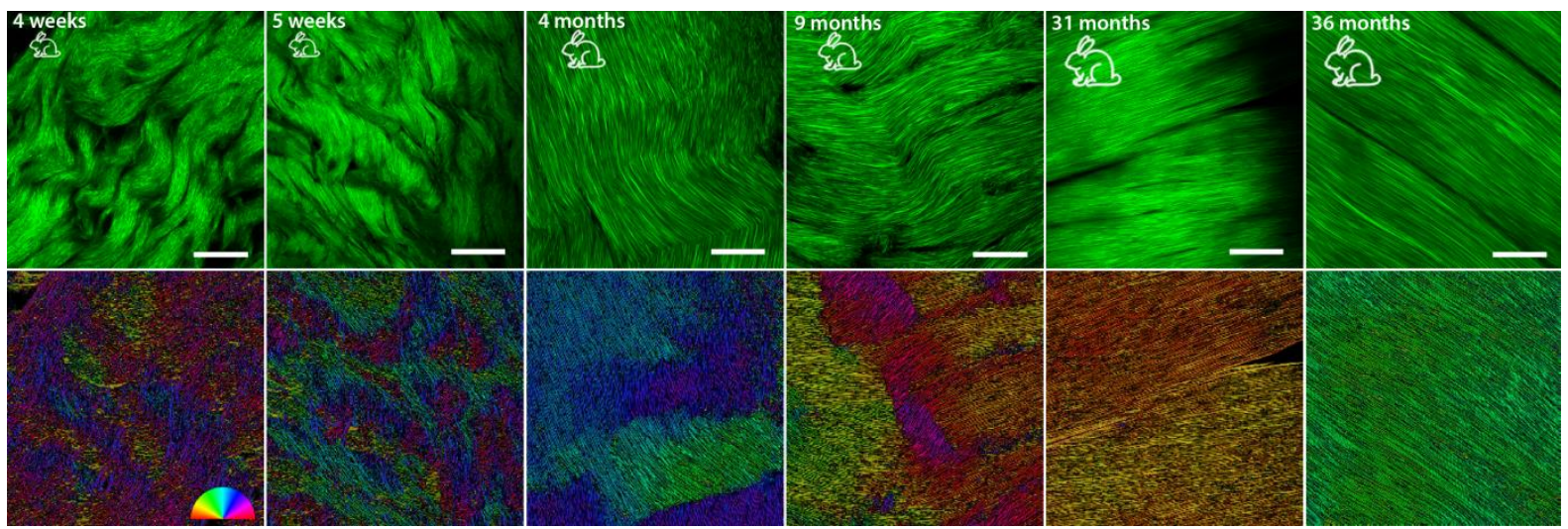

Fig. 2. SHG images of aging rabbit tendon microtome sections and colour coded analysis. Top row: microtome sections displayed differences in the structural pattern among 4-week, 5-week, 4-month, 9-month, 31-month and 36-month-old animals. Bottom row: pseudo-coloured RGB images returned by the fibre orientation regularity analysis of the corresponding top row images. The different colour tone symbolizes the change in orientation of the fibres. The higher the intensity, the more uniform the structural pattern in the $16 \mathrm{x} 16$ pixel analysed window. Scale bar $=25 \mu \mathrm{m}$. 

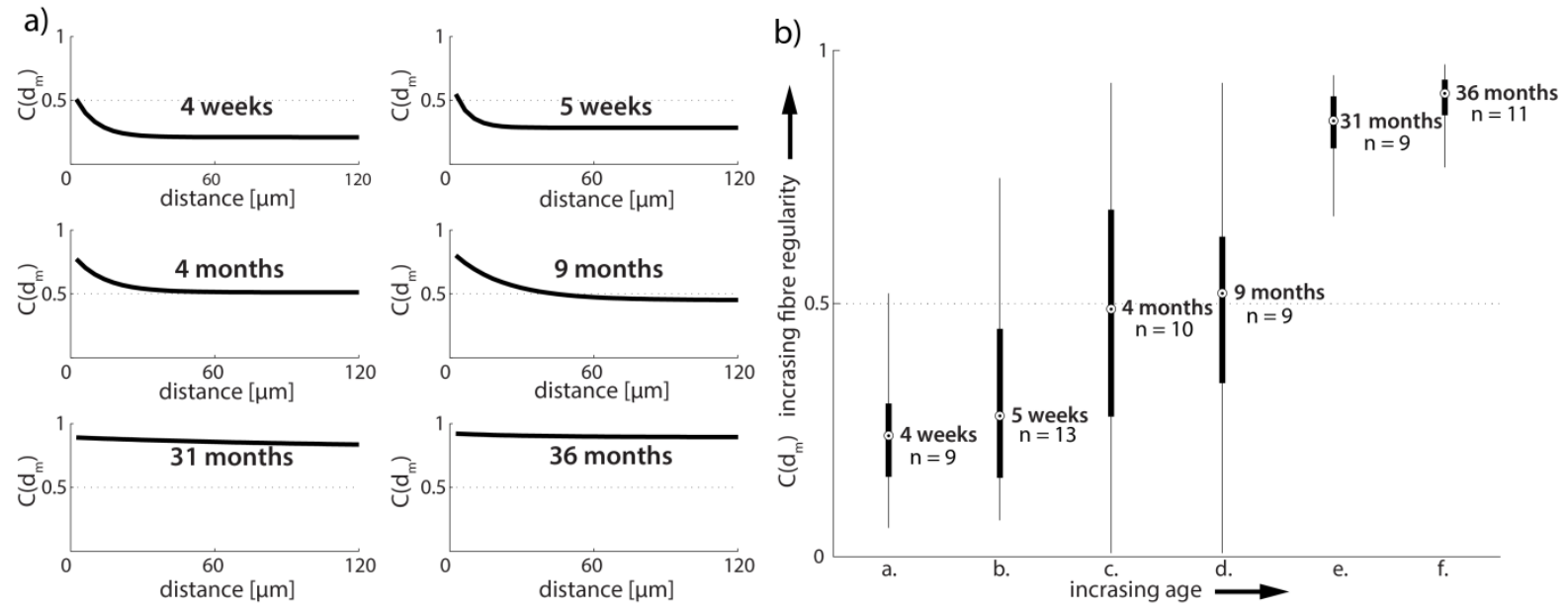

Fig. 3. Orientation regularity correlograms of microtome sections from rabbit tendons. (a) The orientation regularity disappears with distance and drops to lower levels of $C\left(d_{m}\right)$ in the microtome sections from the younger animals. The fibre structures remain aligned with the expected, longitudinal axis of tendon in the sections from the older animals. (b) The main three developmental stages are clearly visible from the box-andwhisker plot. The target marks highlight medians, the black boxes highlight 25th and 75th percentiles and the whiskers highlight maximum/minimum. $n$ is equal to the number of analysed microtome sections per age group.

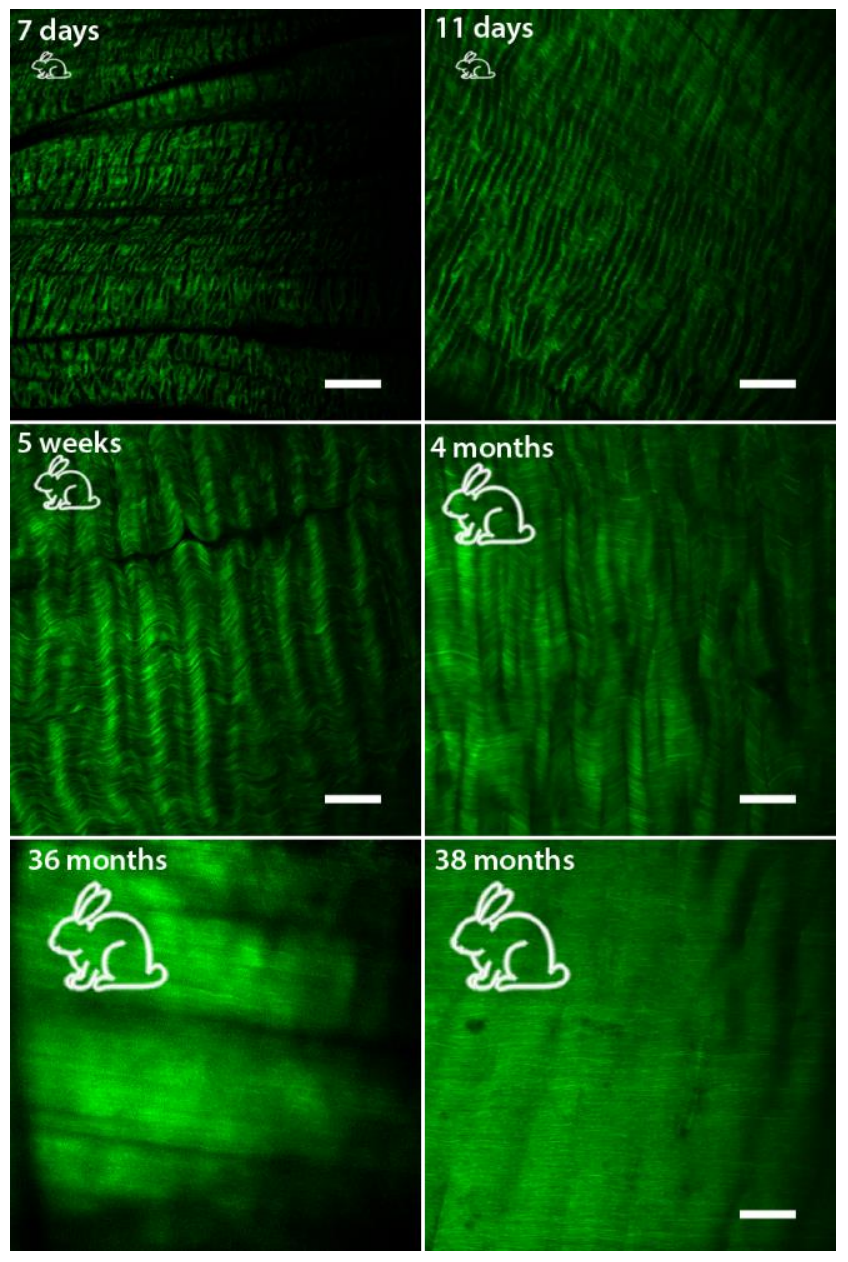


Fig. 4. Images of the crimp pattern in the intact Achilles tendons of rabbits. The global collagen orientation is predominantly arranged parallel to the longitudinal axis of the tendon in the intact samples. This axis is thought to be the main load-bearing axis, and the global orientation is naturally age-independent. In contrast, the local collagen orientation is highly age-dependent. The regular collagen crimp pattern propagates at a certain height/amplitude and periodicity/wavelength through the tendon. Scale bars $=100 \mu \mathrm{m}$.
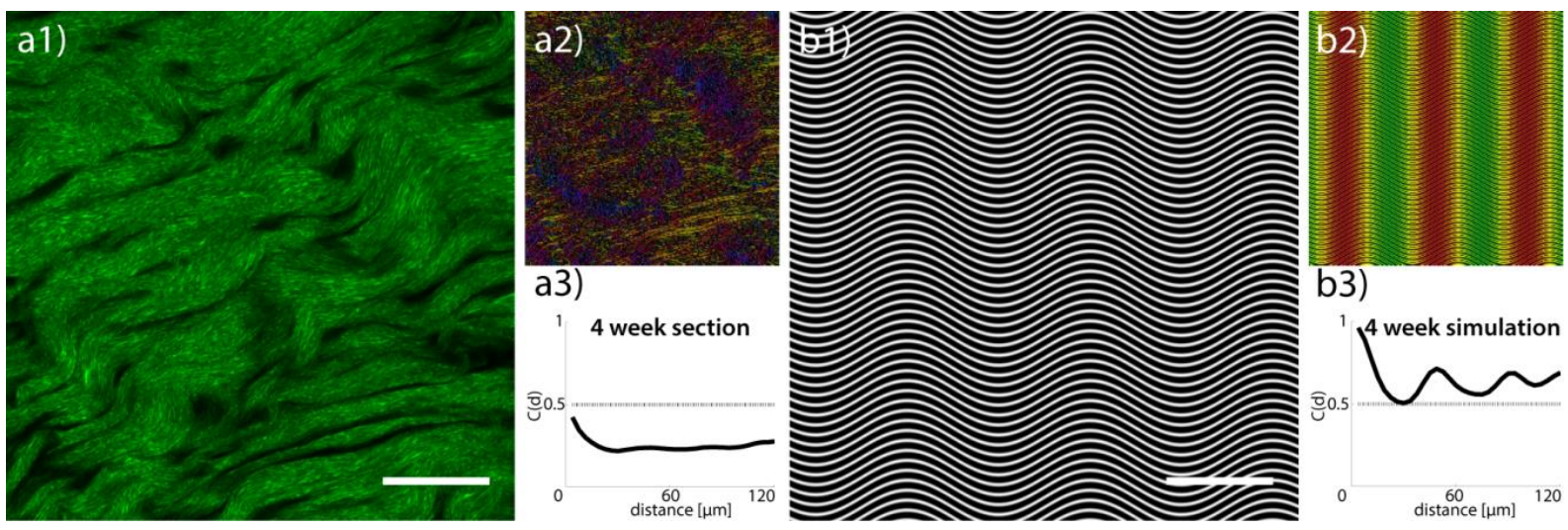

Fig. 5. For 4-week-old animals, the fibres in the microtome section (a1) change randomly and possess a little regularity (a2). On the contrary, the simulation preserves the crimp pattern (b1). The difference is also detectable by comparing the correlograms of real and simulated data $(\mathbf{a 3}, \mathbf{b 3})$. Scale bars $=25 \mu \mathrm{m}$.

Table 1. Quantification of crimp pattern development with age. The development was quantified by the amplitude $A$ and wavelength $\Lambda$.

\begin{tabular}{|c|c|c|c|c|c|c|c|c|c|c|}
\hline Age & 7 days & 11 days & 4 weeks ${ }^{s}$ & 5 weeks & 4 months & 6 months $^{s}$ & 9 months & 18 months $^{\mathrm{s}}$ & 36 months & 38 months \\
\hline Animals & 2 & 1 & 2 & 1 & 1 & 1 & 2 & 1 & 1 & 1 \\
\hline T endons & 4 & 2 & 3 & 2 & 2 & 2 & 3 & 2 & 2 & 2 \\
\hline Crimps & 45 & 154 & 30 & 152 & 70 & 28 & 71 & 18 & 10 & 90 \\
\hline $\mathbf{A}[\mu \mathrm{m}]$ & $2.0 \pm 0.6$ & $2.3 \pm 0.8$ & $4.0 \pm 1.0$ & $6.2 \pm 1.9$ & $9.6 \pm 3.3$ & $6.2 \pm 1.9$ & $5.7 \pm 2.1$ & $4.1 \pm 1.1$ & $2.7 \pm 0.9$ & $3.0 \pm 1.1$ \\
\hline$\Lambda[\mu \mathrm{m}]$ & $19 \pm 4$ & $37 \pm 6$ & $43 \pm 5$ & $71 \pm 13$ & $92 \pm 19$ & $91 \pm 22$ & $103 \pm 23$ & $119 \pm 24$ & $122 \pm 21$ & $122 \pm 32$ \\
\hline
\end{tabular}

${ }^{s}$ The animals were measured by System II. Mean \pm SD.

$\$$ The animals were measured by System II. Mean \pm SD. 